\title{
Erratum to: Multiple driving factors explain spatial and temporal variability in coral calcification rates on the Bermuda platform
}

\author{
A. Venti $\cdot$ A. Andersson $\cdot$ C. Langdon
}

Published online: 18 September 2014

(c) Springer-Verlag Berlin Heidelberg 2014

\section{Erratum to: Coral Reefs}

\section{DOI 10.1007/s00338-014-1191-9}

This erratum corrects errors in Figures 5 and 7 and Tables 1, 2, and 5 of the original publication.

In Table 1, columns 3 [Total linear extension $(\mathrm{mm})$ ] and $5\left[\mathrm{G}\left(\mathrm{g} \mathrm{CaCO}_{3} \mathrm{~cm}^{-2} \mathrm{yr}^{-1}\right)\right]$ are both a factor of 10 less than what is reported. The units $(\mathrm{mm})$ are correct.
In Table 2, columns 3, 4, 5, 7, 8, 9, 10, 11, and 13 are all a factor of 10 less than what is reported. The units $(\mathrm{mm})$ are correct.

The $Y$-axis of Figure $5 \mathrm{a}, \mathrm{b}$ is " $\mathrm{mmol} \mathrm{CaCO}_{3} \mathrm{~m}^{-2} \mathrm{~d}^{-1}$."

Corrected versions of Tables 1 and 2 and Figure 5 are available as Electronic Supplementary Material.

New versions of Fig. 7 and Table 5 are presented here.

The online version of the original article can be found under doi:10.1007/s00338-014-1191-9.

Electronic supplementary material The online version of this article (doi:10.1007/s00338-014-1213-7) contains supplementary material, which is available to authorized users.

\section{A. Venti $(\bowtie) \cdot$ C. Langdon}

Rosenstiel School of Marine and Atmospheric Sciences,

University of Miami, 4600 Rickenbacker CSWY, Miami,

FL 33149, USA

e-mail: aventi@rsmas.miami.edu

C. Langdon

e-mail: clangdon@rsmas.miami.edu

\section{A. Andersson}

Scripps Institution of Oceanography, University of California San Diego, 9500 Gilman Dr., La Jolla, CA 92093-0202, USA

e-mail: aandersson@ucsd.edu 

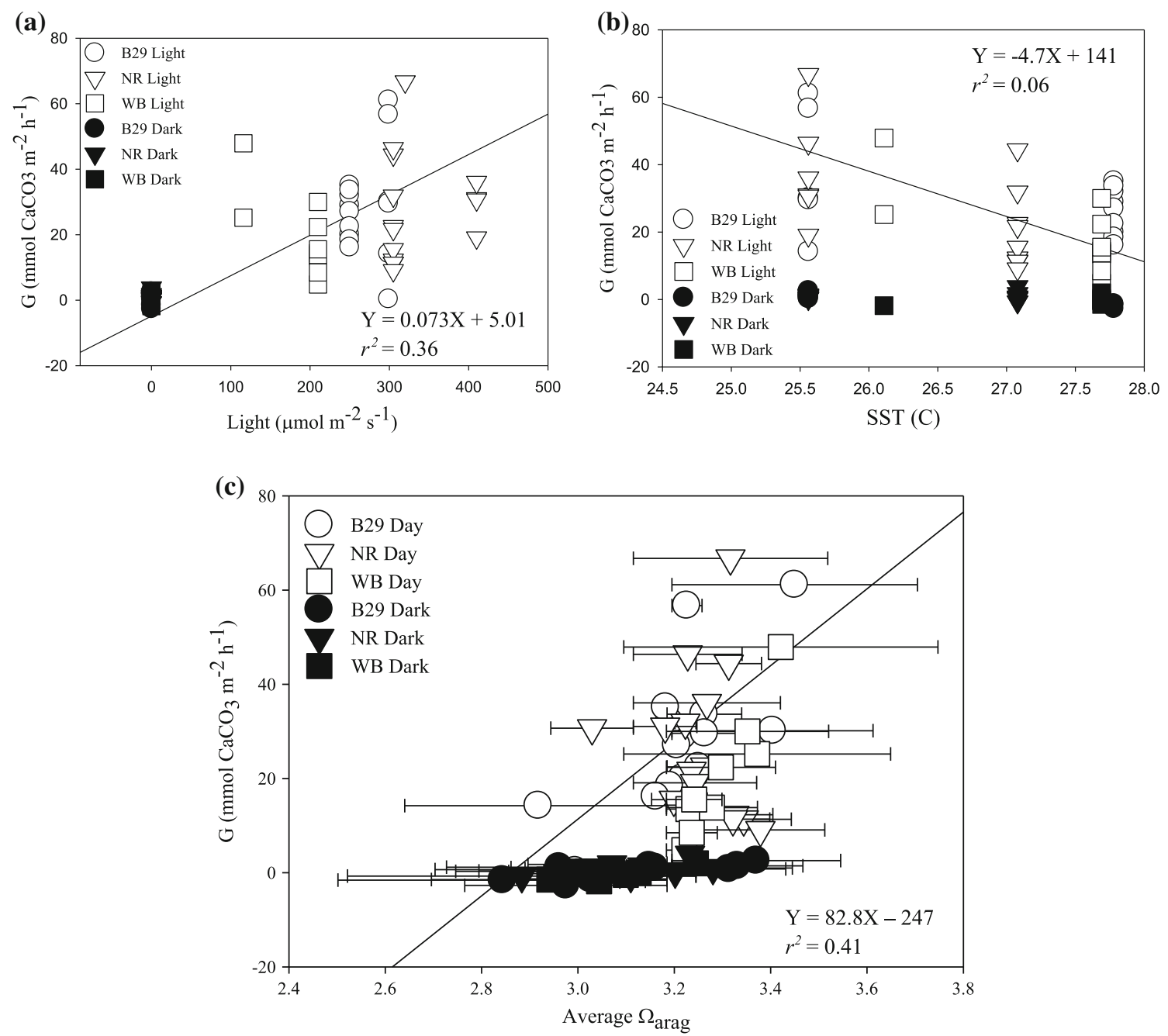

Fig. 7 Hourly coral calcification rates under light (open) and dark (filled) treatments and corresponding a light, b temperature, and c aragonite saturation states within each incubation chamber at three study sites distributed across the northern Bermuda reef platform. Error bars in $\mathbf{c}$ represent the range of aragonite saturation states experienced by each coral during the roughly 1.5 -h incubation period. $\Omega_{\text {arag }}$ is highest at the beginning and decrease throughout the incubation period. Light and temperature remained constant throughout the 1.5 -h incubation 
Table 5 Seasonal coral calcification rates

\begin{tabular}{|c|c|c|c|c|c|c|}
\hline Study & Study site & Method & $\begin{array}{l}\text { Growth } \\
\text { period }\end{array}$ & $\begin{array}{l}\text { Coral } \\
\text { species }\end{array}$ & $\begin{array}{l}\mathrm{G}\left(\mathrm{mmol} \mathrm{CaCO}_{3}\right. \\
\left.\mathrm{m}^{-2} \mathrm{~d}^{-1}\right)\end{array}$ & $\begin{array}{l}\text { Seasonal } \Delta G\left(\mathrm{mmol} \mathrm{CaCO}_{3}\right. \\
\left.\mathrm{m}^{-2} \mathrm{~d}^{-1}\right)\end{array}$ \\
\hline Gladfelter et al. (1978) & St Croix, USVI & Staining & Summer & M. annularis & $355( \pm 24)$ & \\
\hline Gladfelter et al. (1978) & St Croix, USVI & Staining & Winter & M. annularis & $282( \pm 21)$ & 73 \\
\hline Gladfelter et al. (1978) & St Croix, USVI & Staining & Summer & M. annularis & $299( \pm 18)$ & \\
\hline Gladfelter et al. (1978) & St Croix, USVI & Staining & Winter & M. annularis & $380( \pm 18)$ & -81 \\
\hline Gladfelter et al. (1978) & St Croix, USVI & Staining & Summer & P. astreoides & $143( \pm 13)$ & \\
\hline Gladfelter et al. (1978) & St Croix, USVI & Staining & Winter & P. astreoides & $136( \pm 8)$ & 7 \\
\hline Banes and Lough (1993) & Central BGR & X-ray & Summer & Porites & $372( \pm 33)$ & \\
\hline Banes and Lough (1993) & Central BGR & X-ray & Winter & Porites & $323( \pm 32)$ & 49 \\
\hline This study & Bermuda, WB & Staining/X-ray & Summer & P. astreoides & $200( \pm 27)$ & \\
\hline This study & Bermuda, WB & Staining/X-ray & Winter & P. astreoides & $148( \pm 13)$ & 65 \\
\hline This study & Bermuda, B29 & Staining/X-ray & Summer & P. astreoides & $229( \pm 77)$ & \\
\hline This study & Bermuda, B29 & Staining/X-ray & Winter & P. astreoides & $140( \pm 39)$ & 87 \\
\hline This study & Bermuda, NR & Staining/X-ray & Summer & D. strigosa & $293( \pm 22)$ & \\
\hline This study & Bermuda, NR & Staining/X-ray & Winter & D. strigosa & $132( \pm 30)$ & 109 \\
\hline This study & Bermuda, WB & Staining/X-ray & Summer & D. strigosa & $216( \pm 51)$ & \\
\hline This study & Bermuda, WB & Staining/X-ray & Winter & D. strigosa & $118( \pm 13)$ & 93 \\
\hline
\end{tabular}

Calcification rates from Gladfelter et al. (1978) and Barnes and Lough (1993) were estimated using measured seasonal extension rates and assuming an average densities of $1.61 \mathrm{~g} \mathrm{~cm}^{-3}$ for $M$. annularis (Carricart-Ganivet and Merino 2001) and $1.52 \mathrm{~g} \mathrm{~cm}^{-3}$ for $P$. astreoides (Elizalde-Rendón et al. 2010). Though density values change seasonally, coral calcification rates for M. annularis and $P$. astreoides have been shown to be driven by changes in skeletal extension and not density (Cooper et al. 2008; Elizalde-Rendón et al. 2010). Some studies even report no annual change in density for P. astreoides; thus, changes in calcification are driven solely by changes in extension (Tanzil et al. 2009), thus justifying our use of average annual density to estimate seasonal calcification rates. The lack of measured seasonal calcification rate data exemplifies the uniqueness of the present data and the need for more seasonal calcification rate studies 